\title{
Dalpiciclib or placebo plus fulvestrant in hormone receptor-positive and HER2-negative advanced breast cancer: a randomized, phase 3 trial
}

\author{
Binghe Xu®i 1,23凶, Qingyuan Zhang ${ }^{2,23}$, Pin Zhang ${ }^{1,23}$, Xichun $\mathrm{Hu}^{3}$, Wei $\mathrm{Li}^{4}$, Zhongsheng Tong ${ }^{5}$, \\ Tao Sun ${ }^{6}$, Yuee Teng ${ }^{7}$, Xinhong Wu ${ }^{8}$, Quchang Ouyang ${ }^{9}$, Xi Yan ${ }^{10}$, Jing Cheng ${ }^{11}$, Qiang Liu' ${ }^{12}$ \\ Jifeng Feng ${ }^{13}$, Xiaojia Wang ${ }^{14}$, Yongmei Yin ${ }^{15}$, Yanxia Shi ${ }^{16}$, Yueyin Pan ${ }^{17}$, Yongsheng Wang ${ }^{18}$, \\ Weimin Xie ${ }^{19}$, Min Yan ${ }^{20}$, Yunjiang Liu ${ }^{21}$, Ping Yan ${ }^{22}$, Fei Wu ${ }^{22}$, Xiaoyu Zhu ${ }^{22}$, Jianjun Zou ${ }^{22}$ and \\ DAWNA-1 Study Consortium
}

\begin{abstract}
Blockade of the cyclin-dependent kinase 4 and 6 pathway has been shown to be effective in the treatment of hormone receptor-positive advanced breast cancer (ABC). We report the interim results of DAWNA-1 (NCT03927456), a double-blind, randomized, phase 3 trial of dalpiciclib (a new cyclin-dependent kinase 4 and 6 inhibitor) plus fulvestrant in hormone receptor-positive, HER2-negative ABC with disease progression after endocrine therapy. A total of $\mathbf{3 6 1}$ patients were randomized 2:1 to receive dalpiciclib plus fulvestrant or placebo plus fulvestrant. The study met the primary end point, showing significantly prolonged investigator-assessed progression-free survival with dalpiciclib plus fulvestrant versus placebo plus fulvestrant (median $=15.7,95 \%$ confidence interval $(\mathrm{Cl})=11.1-$ not reached versus $7.2,95 \% \mathrm{Cl}=5.6-9.2$ months; hazard ratio $=0.42,95 \% \mathrm{Cl}=0.31-0.58$; one-sided $P<0.0001$ (boundary was $P \leq 0.008$ )). The most common grade 3 or 4 adverse events with dalpiciclib plus fulvestrant were neutropenia (84.2\%) and leukopenia (62.1\%). The incidence of serious adverse events was $5.8 \%$ with dalpiciclib plus fulvestrant versus $6.7 \%$ with placebo plus fulvestrant. Our findings support dalpiciclib plus fulvestrant as a new treatment option for pretreated hormone receptor-positive, HER2-negative ABC.
\end{abstract}

G lobally, breast cancer is the most frequently diagnosed cancer and the leading cause of cancer-related mortality in women ${ }^{1}$. Hormone receptor-positive tumors are the predominant subtype of breast cancer, accounting for $>75 \%$ of all cases $^{2}$. Endocrine therapy is the cornerstone for the treatment of hormone receptor-positive breast cancer $^{3}$; however, acquired resistance develops in nearly all patients ${ }^{4}$. There is a dire need for new therapies that can potentially overcome or at least delay the onset of endocrine resistance.

Cyclin-dependent kinases 4 and 6 (CDK4/6) are key mediators of cell proliferation via the promotion of cell cycle transition from the G1 to the S phase. Hyperactivity of the CDK4/6 pathway is a common feature of hormone receptor-positive breast cancer ${ }^{5}$ and is often implicated in resistance to endocrine therapy ${ }^{6}$. Several phase 3 trials have assessed the usefulness of the combination of a CDK4/6 inhibitor with endocrine therapy in hormone receptor-positive, HER2-negative $\mathrm{ABC}^{7-12}$. The addition of palbociclib, ribociclib or abemaciclib to fulvestrant has been shown to improve survival outcomes in patients who relapsed or progressed on previous endocrine therapy ${ }^{7-9}$, leading to the approval of these combinations by the U.S. Food and Drug Administration and the European Medicines Agency in treating this patient population.

Dalpiciclib (SHR6390) is a new, orally administered, selective CDK4/6 inhibitor ${ }^{13,14}$. In preclinical studies, dalpiciclib was shown to

\footnotetext{
'Department of Medical Oncology and Clinical Trial Center, National Cancer Center/National Clinical Research Center for Cancer/Cancer Hospital, Chinese Academy of Medical Sciences and Peking Union Medical College, Beijing, China. ${ }^{2}$ Department of Medical Oncology, Harbin Medical University Cancer Hospital, Harbin, China. ${ }^{3}$ Department of Medical Oncology, Fudan University Cancer Hospital, Shanghai, China. ${ }^{4}$ Department of Medical Oncology, The First Hospital of Jilin University, Changchun, China. ${ }^{5}$ Department of Medical Oncology, Tianjin Medical University Cancer Institute and Hospital, Tianjin, China. ${ }^{6}$ Department of Medical Oncology, Cancer Hospital of China Medical University/Liaoning Cancer Hospital, Shenyang, China. ${ }^{7}$ Department of Medical Oncology, The First Affiliated Hospital of China Medical University, Shenyang, China. ${ }^{8}$ Department of Breast Oncology, Hubei Cancer Hospital, Wuhan, China. ${ }^{9}$ Department of Medical Oncology, Hunan Cancer Hospital, Changsha, China. ${ }^{10}$ Department of Head and Neck Cancer, West China Hospital, Sichuan University, Chengdu, China. "Department of Breast Oncology, Union Hospital, Tongji Medical College, Huazhong University of Science \& Technology, Wuhan, China. ${ }^{12}$ Department of Medical Oncology, Sun Yat-Sen Memorial Hospital, Sun Yat-Sen University, Guangzhou, China. ${ }^{13}$ Department of Medical Oncology, Jiangsu Cancer Hospital, Nanjing, China. ${ }^{14}$ Department of Medical Oncology, Cancer Hospital of the University of Chinese Academy of Sciences/Zhejiang Cancer Hospital, Institute of Cancer and Basic Medicine, Chinese Academy of Sciences, Hangzhou, China. ${ }^{15}$ Department of Oncology, Jiangsu Province Hospital, Nanjing, China. ${ }^{16}$ Department of Medical Oncology, Sun Yat-Sen University Cancer Center, Guangzhou, China. ${ }^{17}$ Department of Medical Oncology/Chemotherapy, Anhui Provincial Hospital, Hefei, China. ${ }^{18}$ Breast Cancer Center, Shandong Cancer Hospital, Jinan, China. ${ }^{19}$ Department of Breast, Bone \& Soft Tissue Oncology, Guangxi Medical University Cancer Hospital, Nanning, China. ${ }^{20}$ Department of Breast Disease, Henan Breast Cancer Center/The Affiliated Cancer Hospital of Zhengzhou University \& Henan Cancer Hospital, Zhengzhou, China. ${ }^{21}$ Breast Center, The Fourth Hospital of Hebei Medical University, Shijiazhuang, China. ${ }^{22}$ Jiangsu Hengrui Pharmaceuticals Co., Ltd, Shanghai, China. ${ }^{23}$ These authors contributed equally: Binghe $\mathrm{Xu}$, Qingyuan Zhang, Pin Zhang. ${ }^{*} \mathrm{~A}$ list of authors and their affiliations appears at the end of the paper. 凶e-mail: bhxu@hotmail.com
} 


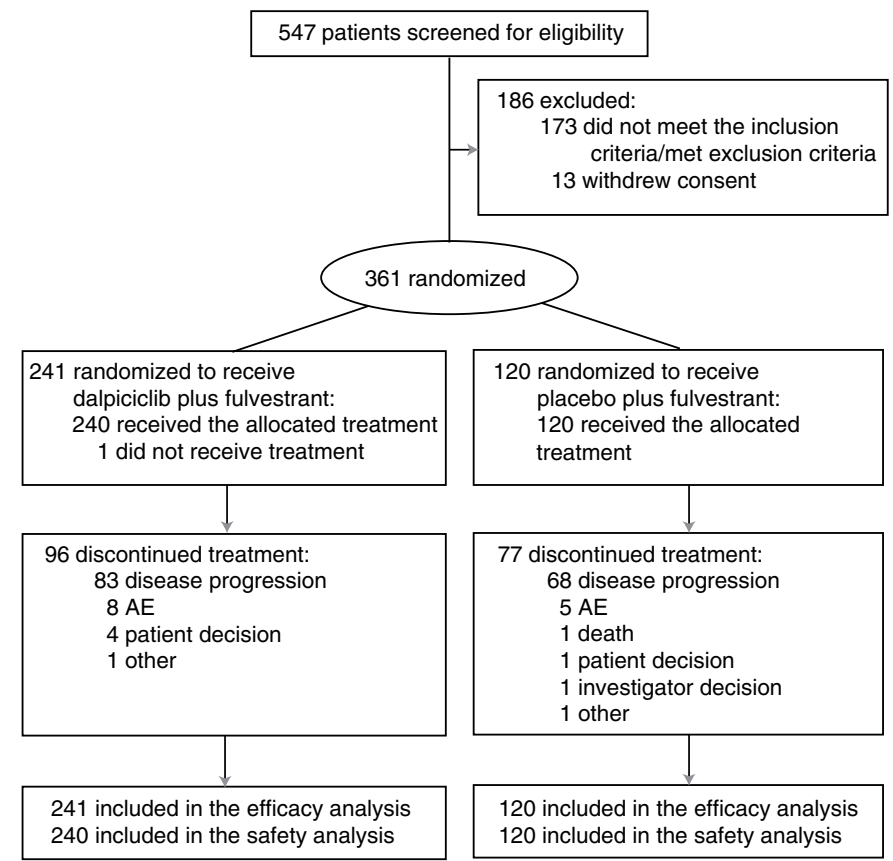

Fig. 1 | Trial profile. The data cutoff date was 15 November 2020.

exert potent antitumor activity (half maximal inhibitory concentration $=12.4 \mathrm{nM}$ for CDK4 and $9.9 \mathrm{nM}$ for CDK6) via retinoblastoma protein-dependent cytostasis ${ }^{13,14}$. Dalpiciclib as monotherapy has demonstrated tolerability and preliminary clinical activity in patients with heavily pretreated hormone receptor-positive, HER2-negative $\mathrm{ABC}^{15}$. In a phase $1 \mathrm{~b}$ trial, we established the synergic effects of dalpiciclib with fulvestrant in the treatment of ABC (NCT03481998; Hengrui data on file). In this study, we conducted a randomized, double-blind, placebo-controlled phase 3 trial to further assess the efficacy and safety of dalpiciclib plus fulvestrant in patients with hormone receptor-positive, HER2-negative ABC who relapsed or progressed on previous endocrine therapy. In this paper, we report the findings of the interim analysis.

\section{Results}

Patient allocation and baseline characteristics. Between 25 June 2019 and 2 September 2020, 547 patients were screened and 361 eligible patients were randomly assigned to receive dalpiciclib plus fulvestrant $(n=241)$ or placebo plus fulvestrant $(n=120)$. All 361 randomized patients were included in the efficacy analysis and 360 treated patients (one patient in the dalpiciclib plus fulvestrant group did not receive treatment) were included in the safety analysis (Fig. 1). As of the data cutoff of the interim analysis (15 November 2020), 144 (59.8\%) of 241 patients in the dalpiciclib plus fulvestrant group and $43(35.8 \%)$ of 120 patients in the placebo plus fulvestrant group were still receiving treatment; the median follow-up was 10.7 months (range $=0.2-16.7$ ) and 10.6 months (range $=0.8-16.7$ ), respectively. Sixty-five $(27.0 \%)$ patients in the dalpiciclib plus fulvestrant group and $49(40.8 \%)$ in the placebo plus fulvestrant group received at least 1 subsequent antitumor therapy after discontinuation of the study treatment (Supplementary Table 1).

Baseline characteristics were generally balanced between the two groups (Table 1). The overall median age was 51.0 years; $55.7 \%$ of patients were postmenopausal women, $60.1 \%$ had visceral metastases and $72.6 \%$ had received one previous line of endocrine therapy.

Efficacy. At the time of the interim analysis, $86(35.7 \%)$ of 241 patients in the dalpiciclib plus fulvestrant group and $76(63.3 \%)$ of 120 patients in the placebo plus fulvestrant group had disease progression or died.
Table 1 | Demographics and baseline characteristics of the study cohort

\begin{tabular}{|cll|} 
& $\begin{array}{l}\text { Dalpiciclib plus } \\
\text { fulvestrant } \\
(\boldsymbol{n}=\mathbf{2 4 1})\end{array}$ & $\begin{array}{l}\text { Placebo plus } \\
\text { fulvestrant } \\
(\boldsymbol{n}=120)\end{array}$ \\
\hline Median age, years (IQR) & $50.7(45.3-59.3)$ & $52.4(45.5-60.6)$ \\
\hline$<65$ years, $n(\%)$ & $211(87.6)$ & $108(90.0)$ \\
$\geq 65$ years, $n(\%)$ & $30(12.4)$ & $12(10.0)$
\end{tabular}

Eastern Cooperative Oncology Group performance status, $n(\%)$

$\begin{array}{lll}0 & 116(48.1) & 46(38.3) \\ 1 & 125(51.9) & 74(61.7)\end{array}$

Menopausal status, $n$ (\%)

$\begin{array}{lll}\text { Postmenopausal } & 135(56.0) & 66(55.0) \\ \begin{array}{l}\text { Premenopausal or } \\ \text { perimenopausal }\end{array} & 106(44.0) & 54(45.0)\end{array}$

perimenopausal

Hormone receptor status, $n$ (\%)

$\begin{array}{lll}\text { ER-positive and PR-positive } & 192(79.7) & 91(75.8) \\ \text { ER-positive and PR-negative } & 46(19.1) & 29(24.2) \\ \text { ER-negative and PR-positive } & 3(1.2) & 0 \\ \text { Measurable disease, } n(\%) & 198(82.2) & 100(83.3)\end{array}$

No. of metastatic sites, $n$ (\%)

$\begin{array}{lll}<4 & 199(82.6) & 98(81.7) \\ \geq 4 & 42(17.4) & 22(18.3)\end{array}$

Visceral metastases, $n(\%)$

$\begin{array}{cll}\text { Yes } & 142(58.9) & 75(62.5) \\ \text { No } & 99(41.1) & 45(37.5) \\ \text { Bone-only } & 42(17.4) & 19(15.8) \\ \text { Other } & 57(23.7) & 26(21.7)\end{array}$

Previous lines of endocrine therapy, $n(\%)$

$1 \quad 175(72.6) \quad 87(72.5)$

2

$66(27.4)$

Previous endocrine therapy, $n$ (\%)

\begin{tabular}{lll} 
SERMs only & $91(37.8)$ & $37(30.8)$ \\
Aromatase inhibitors only & $79(32.8)$ & $51(42.5)$ \\
$\begin{array}{l}\text { SERMs and aromatase } \\
\text { inhibitors }\end{array}$ & $71(29.5)$ & $32(26.7)$ \\
\hline $\begin{array}{l}\text { Prious chemotherapy for } \\
65(27.0)\end{array}$ & $42(35.0)$
\end{tabular}

recurrent/metastatic disease

$E R$, estrogen receptor; $P R$, progesterone receptor; SERM, selective estrogen receptor modulator.

The primary end point of investigator-assessed progression-free survival was significantly prolonged with dalpiciclib plus fulvestrant versus placebo plus fulvestrant (median $=15.7$ months, $95 \%$ $\mathrm{CI}=11.1-$ not reached versus 7.2 months, $95 \% \mathrm{CI}=5.6-9.2$; hazard ratio $(\mathrm{HR})=0.42,95 \% \mathrm{CI}=0.31-0.58$; one-sided $P<0.0001$; Fig. $2 \mathrm{a})$. The study met the primary end point at the interim analysis according to the recommendation from the independent data monitoring committee. The progression-free survival rates at 6 and 12 months in the dalpiciclib plus fulvestrant group and placebo plus fulvestrant group were $76.4 \%(95 \% \mathrm{CI}=70.1-81.5)$ versus $53.2 \%(95 \%$ $\mathrm{CI}=43.5-62.0)$ and $51.8 \%(95 \% \mathrm{CI}=43.2-59.8)$ versus $29.1 \%(95 \%$ $\mathrm{CI}=20.2-38.5)$, respectively. Progression-free survival based on the independent review committee (IRC) assessment was consistent with the investigator-assessed results $(\mathrm{HR}=0.45,95 \% \mathrm{CI}=0.32-0.64$; one-sided $P<0.0001$; Fig. 2b). During the study, 39 (16.2\%) patients in the dalpiciclib plus fulvestrant group and $14(11.7 \%)$ patients in 
$\mathbf{a}$

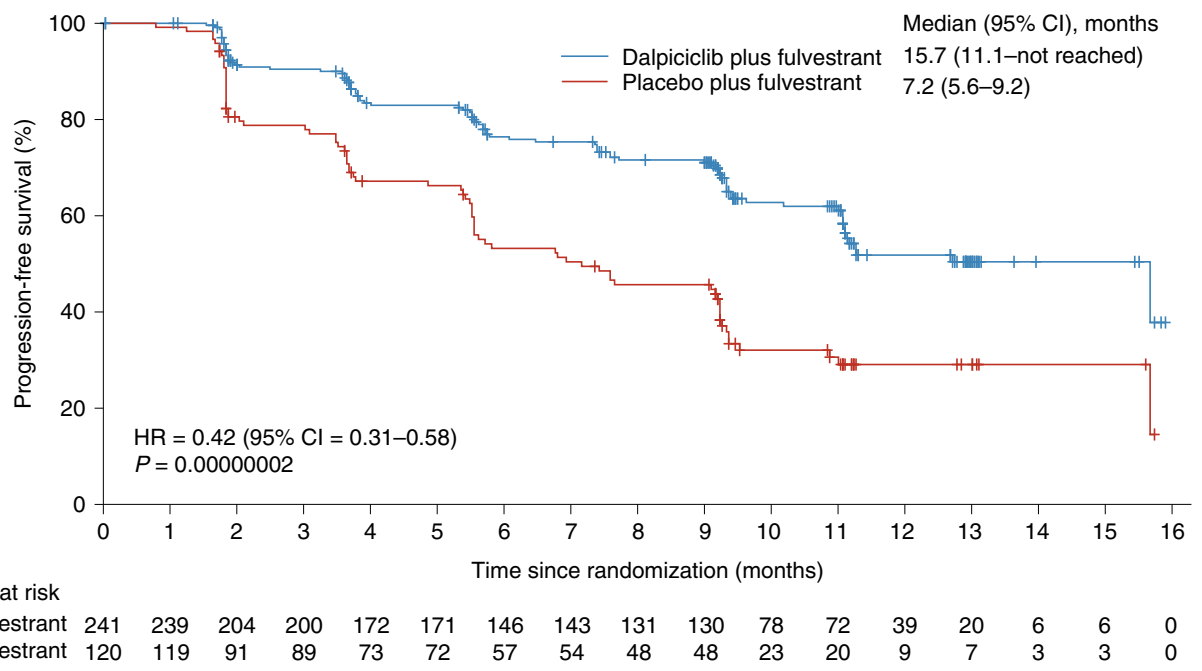

b

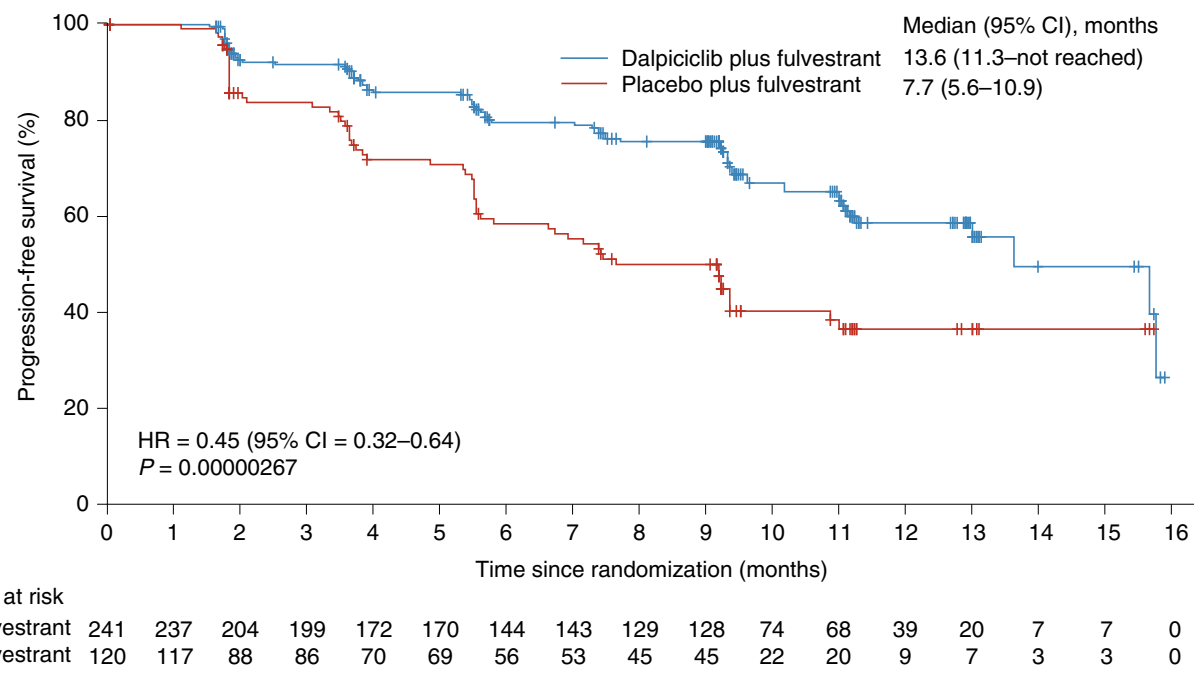

Fig. 2 | Kaplan-Meier analyses of progression-free survival. a,b, Progression-free survival per investigator (primary analysis) (a) and IRC (b). Treatment effects were compared using the stratified log-rank test; HRs and corresponding 95\% Cls were estimated using the stratified Cox proportional hazards model. Stratification factors were the presence of visceral metastasis (yes versus no) and menopausal status (postmenopausal versus premenopausal or perimenopausal). $P$ values are one-sided with no adjustment for multiplicity.

the placebo plus fulvestrant group had $>7$-d delay in at least one treatment component due to the COVID-19 pandemic. Sensitivity analysis with patients censored at the last radiographic assessment before treatment delay yielded similar results as the primary analysis (Supplementary Table 2). Improvement in progression-free survival with dalpiciclib plus fulvestrant was consistent across all prespecified baseline subgroups (Fig. 3) and across patients with different disease-free intervals (post-hoc; Supplementary Table 3) compared with placebo plus fulvestrant (all HRs $<1$ ).

At the time of the analysis, overall survival data were not mature with a total of 25 deaths recorded: 15 (6.2\%) in the dalpiciclib plus fulvestrant group and $10(8.3 \%)$ in the placebo plus fulvestrant group. Fifty-eight (24.1\%) patients in the dalpiciclib plus fulvestrant group and $49(40.8 \%)$ patients in the placebo plus fulvestrant group received subsequent chemotherapy or died; the median time to first subsequent chemotherapy or death was not reached in the dalpiciclib plus fulvestrant group versus 14.2 months (95\% CI $=9.7-$ not reached) in the placebo plus fulvestrant group $(\mathrm{HR}=0.47,95 \%$ $\mathrm{CI}=0.32-0.69$; one-sided $P<0.0001$; Extended Data Fig. 1). The median time to second objective disease progression or death was not reached in either group.
According to the assessment carried out by the investigator, the objective response rate with dalpiciclib plus fulvestrant versus placebo plus fulvestrant was $27.0 \%(95 \% \mathrm{CI}=21.5-33.0)$ versus $20.0 \%$ $(95 \% \mathrm{CI}=13.3-28.3)$ and the clinical benefit rate was $61.0 \%(95 \%$ $\mathrm{CI}=54.5-67.2)$ versus $45.8 \%$ (95\% $\mathrm{CI}=36.7-55.2)$ (Table 2$)$. The IRC-assessed tumor response results were consistent with the investigator assessment (Supplementary Table 4).

Safety. The median duration of treatment was 9.4 months (interquartile range $(\mathrm{IQR})=4.3-11.4)$ for dalpiciclib and 9.9 months (IQR = 4.7-11.9) for fulvestrant in the dalpiciclib plus fulvestrant group and 5.8 months $(\mathrm{IQR}=3.1-10.4)$ for placebo and 6.1 months (IQR =3.7-11.0) for fulvestrant in the placebo plus fulvestrant group (Supplementary Table 5). Grade 3 or 4 adverse events (AEs) were reported in $212(88.3 \%)$ of 240 patients treated with dalpiciclib plus fulvestrant and 16 (13.3\%) of 120 patients treated with placebo plus fulvestrant. AEs led to dose reduction of dalpiciclib in 67 (27.9\%) patients in the dalpiciclib plus fulvestrant group and of placebo in $2(1.7 \%)$ patients in the placebo plus fulvestrant group; the number of patients who discontinued any treatment component due to AEs was $6(2.5 \%)$ and 4 (3.3\%), respectively (Supplementary 


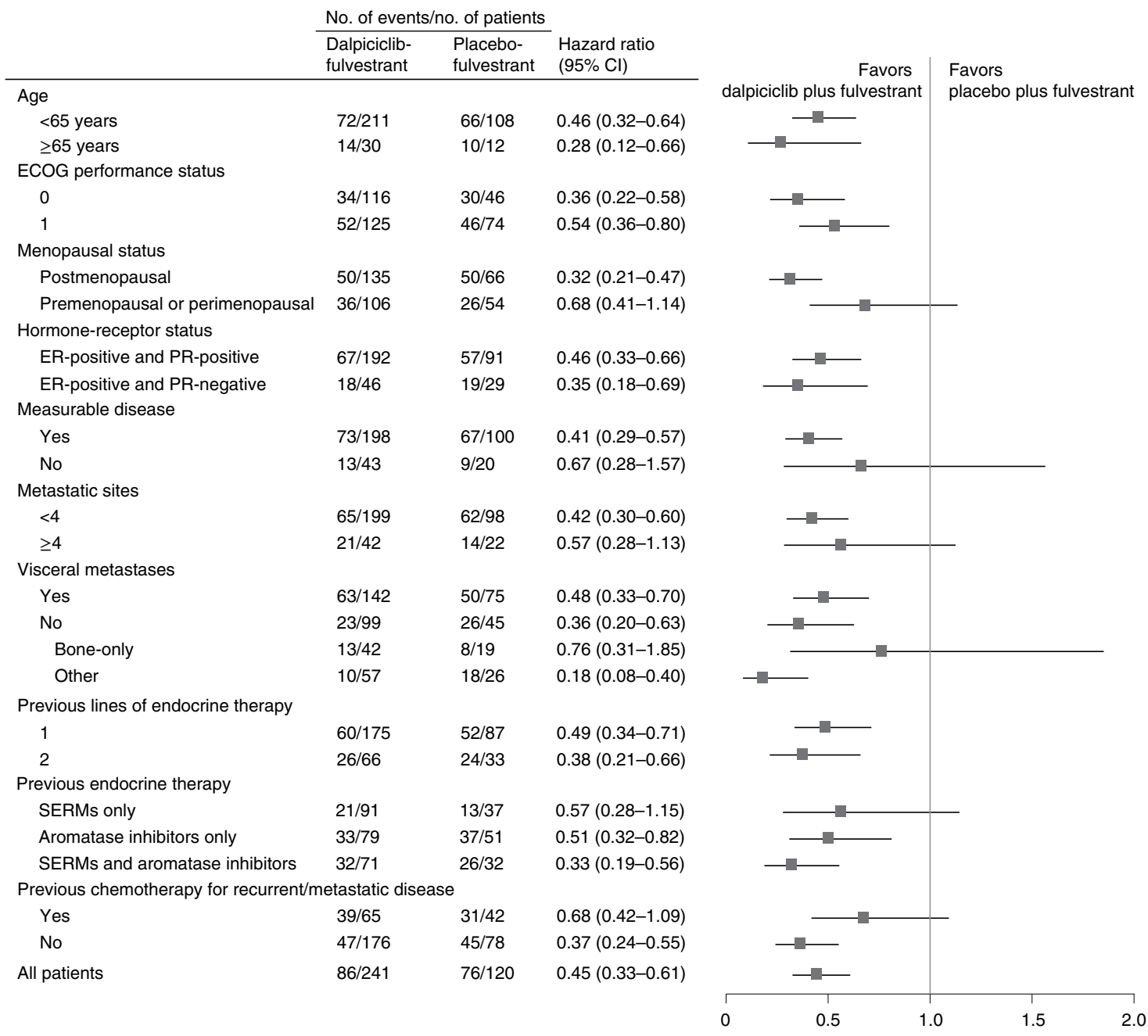

Fig. 3 | Investigator-assessed progression-free survival in prespecified subgroups. HRs and corresponding $95 \% \mathrm{Cls}$ were estimated using the unstratified Cox proportional hazards model.

Table 6). Serious AEs were reported in $14(5.8 \%)$ patients in the dalpiciclib plus fulvestrant group and $8(6.7 \%)$ in the placebo plus fulvestrant group (Supplementary Table 7); of these, 2 cases (bronchitis $(n=1)$, pneumonia $(n=1))$ in the dalpiciclib plus fulvestrant group and 1 case (cryptococcal meningitis) in the placebo plus fulvestrant group were infection-related. Fatal AEs were reported for $2(0.8 \%)$ patients in the dalpiciclib plus fulvestrant group and 4 $(3.3 \%)$ in the placebo plus fulvestrant group (Supplementary Table 8 ); of these, 1 case each in the dalpiciclib plus fulvestrant group (hepatic/respiratory failure) and placebo plus fulvestrant group (death of unknown cause) were possibly related to treatment as assessed by the investigator.

All grade 3 or 4 AEs occurring in $\geq 3 \%$ of patients in either group were hematological toxicities (Table 3 ). The most common grade 3 or 4 AEs after dalpiciclib plus fulvestrant treatment were neutropenia (202 patients $(84.2 \%$ ) versus 0 with placebo plus fulvestrant), leukopenia $(149(62.1 \%)$ versus 0$)$, thrombocytopenia (14 $(5.8 \%)$ versus $1(0.8 \%))$ and lymphopenia $(10(4.2 \%)$ versus 0$)$. In the dalpiciclib plus fulvestrant group, grade 3 (156 patients $(65.0 \%)$ ) or 4 neutropenia (46 (19.2\%)) first onset occurred mostly during the first cycle of treatment (Supplementary Table 9); median duration per grade 3 or 4 event was $3 \mathrm{~d}(\mathrm{IQR}=2.0-4.0)$. The incidence and severity of neutropenia showed a decreasing trend with an increasing number of treatment cycles (Extended Data Fig. 2). From cycle 3 onward, the incidence of grade 4 neutropenia was $\leq 3.9 \%$ (cycle 1 ,
$9.2 \%$; cycle $2,4.6 \%$ ) in all subsequent cycles in the dalpiciclib plus fulvestrant group. There were no cases of febrile neutropenia or treatment discontinuation due to neutropenia.

The most common non-hematological AEs were low-grade liver enzyme abnormalities, with comparable incidences reported for both groups (Table 3). Prolonged QT on electrocardiogram occurred in $16(6.7 \% ; 12(5.0 \%)$ grade $1,2(0.8 \%)$ grade 2 and 2 $(0.8 \%)$ grade 3$)$ of 240 patients in the dalpiciclib plus fulvestrant group and $1(0.8 \%$; grade 3$)$ of 120 patients in the placebo plus fulvestrant group. None of the prolonged QT events led to treatment discontinuation. Thrombotic events were reported for $3(1.3 \%$; grade $1(n=2)$, grade $2(n=1))$ patients in the dalpiciclib plus fulvestrant group and $1(0.8 \%$; grade 2$)$ patient in the placebo plus fulvestrant group.

\section{Discussion}

Our study met its primary end point at the interim analysis by demonstrating that dalpiciclib plus fulvestrant significantly improved progression-free survival versus placebo plus fulvestrant and reduced the risk of disease progression or death by $58 \%(\mathrm{HR}=0.42$, $95 \% \mathrm{CI}=0.31-0.58)$ in patients with hormone receptor-positive, HER2-negative ABC that relapsed or progressed on previous endocrine therapy. While overall survival data are not mature yet, benefit beyond initial study treatment was shown for dalpiciclib over placebo based on time to first subsequent chemotherapy. 
Table 2 | Tumor response per investigator

\begin{tabular}{|c|c|c|c|c|c|}
\hline & $\begin{array}{l}\text { Dalpiciclib plus fulvestrant } \\
(n=241)\end{array}$ & $95 \% \mathrm{Cl}$ & $\begin{array}{l}\text { Placebo plus fulvestrant } \\
(n=120)\end{array}$ & $95 \% \mathrm{Cl}$ & $P$ \\
\hline \multicolumn{6}{|l|}{ Best overall response, $n(\%)$} \\
\hline Complete response & $2(0.8)$ & - & 0 & - & - \\
\hline Partial response & $63(26.1)$ & - & $24(20.0)$ & - & - \\
\hline Stable disease & $149(61.8)$ & - & $70(58.3)$ & - & - \\
\hline Progressive disease & $23(9.5)$ & - & $23(19.2)$ & - & - \\
\hline Objective response rate, $n(\%)$ & $65(27.0)$ & $21.5-33.0$ & $24(20.0)$ & $13.3-28.3$ & 0.0727 \\
\hline Median time to response, months (IQR) & $3.7(1.8-4.0)$ & - & $2.0(1.8-4.7)$ & - & - \\
\hline Median duration of response, months (range) & Not reached (1.7-12.1+) & - & $7.7(1.8-13.9+)$ & - & - \\
\hline Clinical benefit rate, $n(\%)^{a}$ & $147(61.0)$ & $54.5-67.2$ & $55(45.8)$ & $36.7-55.2$ & 0.0033 \\
\hline
\end{tabular}

${ }^{a}$ Defined as the proportion of patients with a complete or partial response or stable disease lasting at least 24 weeks. The between-group comparisons for objective response and clinical benefit rates were implemented using the stratified Cochran-Mantel-Haenszel method. Stratification factors were the presence of visceral metastasis (yes versus no) and menopausal status (postmenopausal versus premenopausal or perimenopausal). $P$ values are one-sided with no adjustment for multiplicity.

Table 3 | Most frequently reported AEs

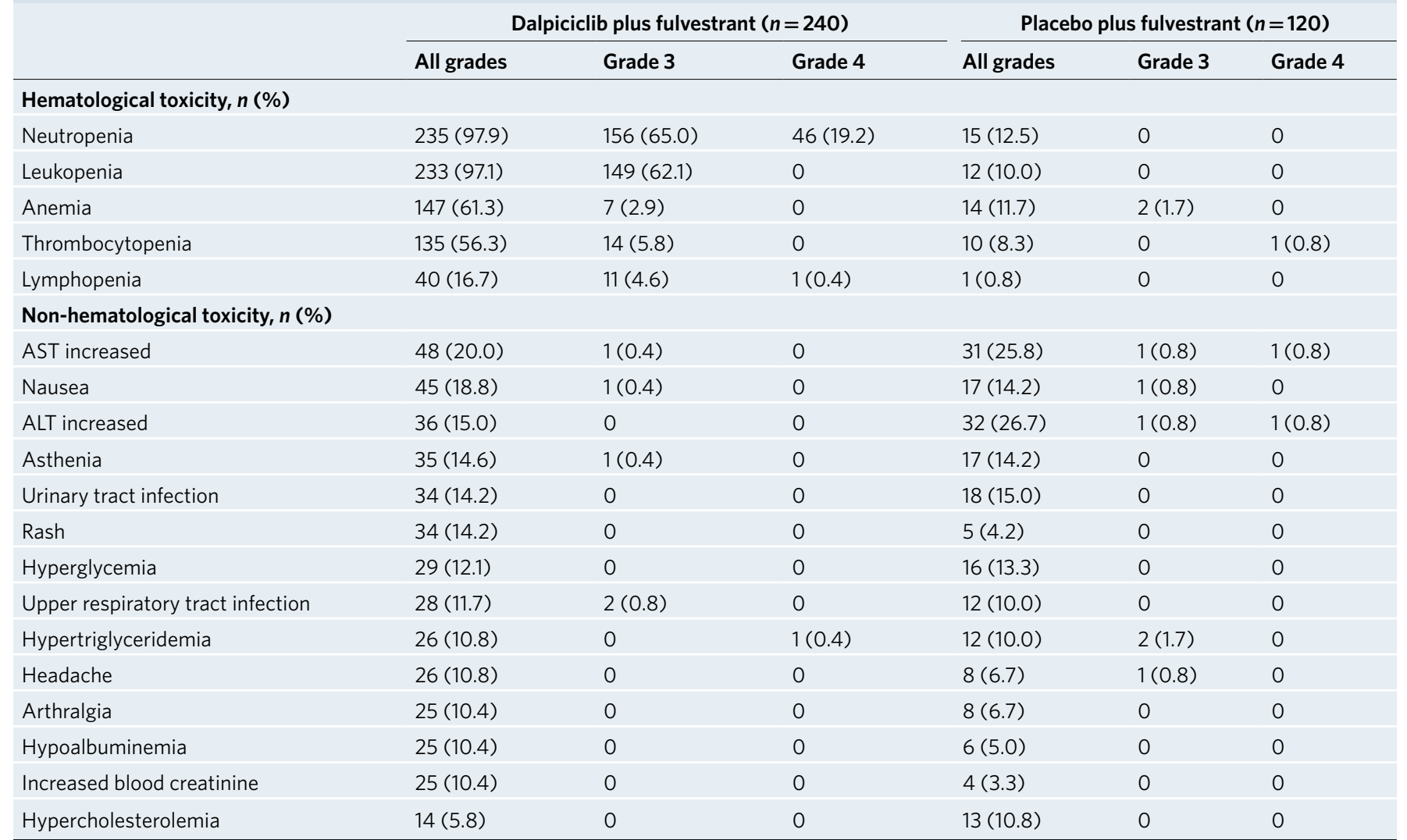

One patient in the dalpiciclib plus fulvestrant group who did not receive treatment was excluded from the safety analysis. Any grade AEs reported in at least $10 \%$ of patients in either group or grade 3 or 4 events reported in at least $3 \%$ are listed. ALT, alanine aminotransferase; AST, aspartate aminotransferase.

The effects of CDK4/6 inhibitors in combination with fulvestrant in patients with hormone receptor-positive, HER2-negative $\mathrm{ABC}$ progressing on previous endocrine therapy have been studied in three global randomized phase 3 trials, PALOMA-3 (palbociclib), MONALEESA-3 (ribociclib) and MONARCH-2 (abemaciclib) ${ }^{7-12}$. These studies differed in some key patient eligibility criteria, particularly regarding the lines of previous endocrine therapy (unlimited in PALOMA-3 and up to 1 in MONALEESA-3/MONARCH-2) and chemotherapy for advanced disease (up to 1 in PALOMA-3 and not permitted in MONALEESA-3/MONARCH-2); however, all trials demonstrated significantly prolonged progression-free survival with the addition of a CDK4/6 inhibitor. Our study patients represented a moderately pretreated population (approximately $70 \%$ with 1 previous line of endocrine therapy, approximately $30 \%$ with previous chemotherapy for advanced disease) and the median progression-free survival in the placebo plus fulvestrant group was 7.2 months compared with 4.6 to 12.8 months in the placebo plus fulvestrant groups in trials of other CDK4/6 inhibitors ${ }^{7-12}$. The relative risk for progression 
or death was $58 \%$ lower with the addition of dalpiciclib, which is comparable to the reduced risk of $54 \%$ with palbociclib, $41 \%$ with ribociclib and $45 \%$ with abemaciclib ${ }^{10-12}$. In line with previous reports for other approved CDK4/6 inhibitors ${ }^{7-9}$, the benefits with dalpiciclib for progression-free survival were consistent regardless of clinicopathological features. Additionally, a numerically higher proportion of patients treated with dalpiciclib plus fulvestrant had an objective response and clinical benefit than those who received placebo plus fulvestrant. One important consideration in the treatment of hormone receptor-positive $\mathrm{ABC}$ was to delay the use of chemotherapy, thus maintaining the quality of life of patients. The risk of receiving first subsequent chemotherapy or death was $53 \%$ lower with dalpiciclib compared with placebo in combination with fulvestrant, providing evidence for extended benefits beyond initial treatment.

The safety profile of dalpiciclib plus fulvestrant was generally tolerable. The most common grade 3 or 4 events associated with dalpiciclib treatment were hematological toxicities (primarily neutropenia). This toxicity profile of dalpiciclib was consistent with CDK4/6 inhibitors with comparable potencies against CDK6 and CDK4 (palbociclib and ribociclib); on the other hand, gastrointestinal toxicity, a common adverse effect of abemaciclib, was rarely observed with dalpiciclib ${ }^{7-12,15}$. Neutropenia was effectively managed with the use of dose modifications and granulocyte colony-stimulating factor, resulting in a median duration of $3 \mathrm{~d}$ per grade 3 or 4 event and no treatment discontinuation due to neutropenia. In addition, there were no cases of febrile neutropenia and the rate of infection-related serious AEs was low (0.8\%). Our results were also in line with previous subgroup analysis that the incidence of neutropenia was slightly higher in Asian patients receiving CDK4/6 inhibitors; however, infective complications were uncommon and tolerability was generally similar to non-Asian patients ${ }^{16,17}$. Notably, $6.7 \%$ of patients in the dalpiciclib group experienced QT prolongation; however, most cases resolved spontaneously without causing clinical symptoms.

The study has some limitations. First, only patients with hormone receptor-positive, HER2-negative $\mathrm{ABC}$ and secondary endocrine resistance were enrolled. The role of dalpiciclib combination therapy in the treatment of patients with primary endocrine resistance requires further investigation. Second, the study was conducted in Chinese patients and the efficacy and tolerability of dalpiciclib plus fulvestrant in other populations is to be established. Third, overall survival data are not mature yet. The long-term survival benefits of adding dalpiciclib to fulvestrant will be further elucidated in our final analysis of the trial.

This phase 3 trial showed that adding dalpiciclib to fulvestrant significantly prolonged progression-free survival, with a manageable safety profile. These findings support the application of dalpiciclib plus fulvestrant as a new treatment option for patients with hormone receptor-positive, HER2-negative $\mathrm{ABC}$ who relapse or progress on previous endocrine therapy.

\section{Online content}

Any methods, additional references, Nature Research reporting summaries, source data, extended data, supplementary information, acknowledgements, peer review information; details of author contributions and competing interests; and statements of data and code availability are available at https://doi.org/10.1038/ s41591-021-01562-9.

Received: 20 July 2021; Accepted: 28 September 2021; Published online: 4 November 2021

\section{References}

1. Sung, H. et al. Global cancer statistics 2020: GLOBOCAN estimates of incidence and mortality worldwide for 36 cancers in 185 countries. $C A$ Cancer J. Clin. 71, 209-249 (2021).

2. Howlader, N. et al. US incidence of breast cancer subtypes defined by joint hormone receptor and HER2 status. J. Natl Cancer Inst. 106, dju055 (2014).

3. Rugo, H. S. et al. Endocrine therapy for hormone receptor-positive metastatic breast cancer: American Society of Clinical Oncology guideline. J. Clin. Oncol. 34, 3069-3103 (2016)

4. Osborne, C. K. \& Schiff, R. Mechanisms of endocrine resistance in breast cancer. Annu. Rev. Med. 62, 233-247 (2011).

5. Bosco, E. E. \& Knudsen, E. S. RB in breast cancer: at the crossroads of tumorigenesis and treatment. Cell Cycle 6, 667-671 (2007).

6. Thangavel, C. et al. Therapeutically activating RB: reestablishing cell cycle control in endocrine therapy-resistant breast cancer. Endocr. Relat. Cancer 18, 333-345 (2011)

7. Turner, N. C. et al. Palbociclib in hormone-receptor-positive advanced breast cancer. N. Engl. J. Med. 373, 209-219 (2015).

8. Slamon, D. J. et al. Phase III randomized study of ribociclib and fulvestrant in hormone receptor-positive, human epidermal growth factor receptor 2-negative advanced breast cancer: MONALEESA-3. J. Clin. Oncol. 36, 2465-2472 (2018).

9. Sledge, G. W. Jr. et al. MONARCH 2: abemaciclib in combination with fulvestrant in women with $\mathrm{HR}^{+} / \mathrm{HER} 2^{-}$advanced breast cancer who had progressed while receiving endocrine therapy. J. Clin. Oncol. 35, 2875-2884 (2017).

10. Turner, N. C. et al. Overall survival with palbociclib and fulvestrant in advanced breast cancer. N. Engl. J. Med. 379, 1926-1936 (2018).

11. Sledge, G. W. Jr. et al. The effect of abemaciclib plus fulvestrant on overall survival in hormone receptor-positive, ERBB2-negative breast cancer that progressed on endocrine therapy--MONARCH 2: a randomized clinical trial. JAMA Oncol. 6, 116-124 (2020).

12. Slamon, D. J. et al. Overall survival with ribociclib plus fulvestrant in advanced breast cancer. N. Engl. J. Med. 382, 514-524 (2020).

13. Long, F. et al. Preclinical characterization of SHR6390, a novel CDK 4/6 inhibitor, in vitro and in human tumor xenograft models. Cancer Sci. 110, 1420-1430 (2019).

14. Wang, J. et al. CDK4/6 inhibitor-SHR6390 exerts potent antitumor activity in esophageal squamous cell carcinoma by inhibiting phosphorylated $\mathrm{Rb}$ and inducing G1 cell cycle arrest. J. Transl. Med. 15, 127 (2017).

15. Zhang, P. et al. A phase 1 study of dalpiciclib, a cyclin-dependent kinase $4 / 6$ inhibitor in Chinese patients with advanced breast cancer. Biomark. Res. 9, 24 (2021).

16. Iwata, H. et al. PALOMA-3: phase III trial of fulvestrant with or without palbociclib in premenopausal and postmenopausal women with hormone receptor-positive, human epidermal growth factor receptor 2-negative metastatic breast cancer that progressed on prior endocrine therapy--safety and efficacy in Asian patients. J. Glob. Oncol. 3, 289-303 (2017).

17. Yap, Y. S. et al. First-line ribociclib 1 letrozole in postmenopausal Asian women with hormone receptor-positive (HR1), human epidermal growth factor receptor 2-negative (HER2-) advanced breast cancer (ABC): a subgroup analysis from MONALEESA-2. Ann. Oncol. 27, IX190 (2016).

Publisher's note Springer Nature remains neutral with regard to jurisdictional claims in published maps and institutional affiliations.

(C) The Author(s), under exclusive licence to Springer Nature America, Inc. 2021

\title{
DAWNA-1 Study Consortium
}

\author{
Binghe $\mathrm{Xu}^{1,23}$, Qingyuan Zhang ${ }^{2,23}$, Pin Zhang ${ }^{1,23}$, Xichun $\mathrm{Hu}^{3}$, Wei $\mathrm{Li}^{4}$, Zhongsheng Tong ${ }^{5}$, Tao Sun ${ }^{6}$, \\ Yuee Teng ${ }^{7}$, Xinhong $\mathbf{W u}^{8}$, Quchang Ouyang ${ }^{9}$, Xi Yan ${ }^{10}$, Jing Cheng ${ }^{11}$, Qiang Liu ${ }^{12}$, Jifeng Feng ${ }^{13}$, \\ Xiaojia Wang ${ }^{14}$, Yongmei Yin ${ }^{15}$, Yanxia Shi ${ }^{16}$, Yueyin Pan ${ }^{17}$, Yongsheng Wang ${ }^{18}$, Weimin Xie ${ }^{19}$, \\ Min Yan ${ }^{20}$, Yunjiang Liu ${ }^{21}$, Ping Yan ${ }^{22}$, Fei Wu ${ }^{22}$, Xiaoyu Zhu ${ }^{22}$ and Jianjun Zou ${ }^{22}$
}




\section{Methods}

Patients. DAWNA-1 (ClinicalTrials.gov ID: NCT03927456) was a multicenter, randomized, double-blind, placebo-controlled, phase 3 trial conducted in 39 centers in China (see Supplementary Information for the complete list of centers). Eligible patients were women of any menopausal status aged 18-75 years with pathologically confirmed hormone receptor-positive, HER2-negative locally advanced or metastatic breast cancer not amenable to curative resection or radiotherapy and not indicated for chemotherapy. Patients must have had radiographically confirmed relapse or disease progression during or after previous endocrine therapy, defined as progression $\geq 6$ months after initiating endocrine therapy for recurrent or metastatic disease or relapse or progression while on adjuvant endocrine therapy but after the first 2 years or within 12 months from completion of adjuvant endocrine therapy. Patients must not have received more than one previous chemotherapy for advanced disease. Additional inclusion criteria included an Eastern Cooperative Oncology Group performance status of 0 or 1, measurable lesion per response evaluation criteria in solid tumors (RECIST) v.1.1 or nonmeasurable bone-only lytic or mixed lesions, adequate organ and bone marrow function and a QT interval corrected for heart rate according to Fridericia's formula of $<470 \mathrm{~ms}$. The key exclusion criteria included brain metastases, previous exposure to fulvestrant, everolimus or other CDK4/6 inhibitor, an important cardiac event within six months or serious infection within four weeks from the first dose. The study protocol is provided in the supplementary files.

The trial was designed and conducted in accordance with good clinical practice guidelines and the Declaration of Helsinki (version 2013). The study protocol and all amendments were reviewed and approved by the independent ethics committee or institutional review board at each site. All patients provided written informed consent before enrollment. Patients were offered minor compensation for their participation in the study (that is, travel costs). An independent data monitoring committee reviewed safety data at regular intervals.

Study design and treatment. Eligible patients were randomized in a 2:1 ratio to receive dalpiciclib plus fulvestrant or placebo plus fulvestrant using a central interactive web response system. Stratification factors were the presence of visceral metastasis (yes versus no) and menopausal status (postmenopausal versus premenopausal or perimenopausal). The randomization sequence was generated by the sponsor's randomization specialist. Investigators registered patients and assigned them based on the randomization sequence obtained from the web response system. Patients, investigators, study site staff and sponsor were masked to treatment assignment. Dalpiciclib $150 \mathrm{mg}$ or matching placebo (identically packaged) was given orally once daily for 3 weeks, followed by 1 week off in each 4 -week cycle. Fulvestrant $500 \mathrm{mg}$ was administered intramuscularly on day 1 and day 15 of the first cycle and then on day 1 of each subsequent 4-week cycle. All premenopausal or perimenopausal women were required to receive a gonadotropin-releasing hormone analog (preferably goserelin) during the study, starting at least $14 \mathrm{~d}$ before the first dose of the study medication. Follicle-stimulating hormone and estradiol levels were measured locally at baseline (mandatory, except for patients who were aged $>60$ years or had bilateral oophorectomy) and then every 4 cycles at the investigator's discretion. Treatment continued until disease progression, unacceptable toxicity, consent withdrawal or investigator's decision. Crossover to dalpiciclib was not allowed for patients progressing on placebo plus fulvestrant. Dose interruptions and reductions (up to 2 in 25-mg decrements) for dalpiciclib or placebo were permitted. Fulvestrant was allowed to be withheld but not reduced in dose. Primary prophylaxis for neutropenia using granulocyte colony-stimulating factor was not allowed as per protocol.

End points and assessment. The primary end point was investigator-assessed progression-free survival, defined as the time of randomization to the first progression per RECIST v.1.1 or death from any cause, whichever occurred first. Secondary end points included overall survival, progression-free survival per IRC, objective response rate, clinical benefit rate, duration of response, time to response, time to first subsequent chemotherapy or death, time to second objective disease progression (defined as the time from randomization to discontinuation of next-line treatment or tumor progression on next-line treatment or death) and safety.

Tumor evaluation was performed at screening, every 8 weeks for the first 56 weeks and every 12 weeks thereafter using computed tomography or magnetic resonance imaging by the investigator and IRC according to RECIST v.1.1. Complete or partial response was required to be confirmed with a subsequent scan at least four weeks after the initial documentation. Safety was monitored from the time of informed consent to $28 \mathrm{~d}$ after the last study dose and was assessed with AEs, laboratory examinations and 12-lead electrocardiograms. AEs were graded according to the Common Terminology Criteria for AEs v.5.0.

Statistical analysis. Assumptions for sample size calculation included a median progression-free survival of 6 months with placebo plus fulvestrant and 10 months with dalpiciclib plus fulvestrant and an HR of 0.60 , a randomization ratio of 2:1 between two groups, an accrual period of 14 months, a study duration of 24 months and a dropout rate of $15 \%$. With a one-sided significance level of $0.025,184$ progression-free survival events were needed to ensure a $90 \%$ power to detect the superiority of dalpiciclib plus fulvestrant over placebo plus fulvestrant. A sample size of 288 patients was required. With the outbreak of the COVID-19 pandemic, the planned sample size was increased to 357 and the sample size for planned events of progression or death was increased to 227 based on the recommendations from the independent data monitoring committee.

An interim analysis was planned when approximately $70 \%$ of the total events of progression or death had occurred. As of 15 November 2020, 162 events (71.4\% of the total projected) had occurred and the corresponding superiority boundary was calculated as one-sided $P \leq 0.0080$ with the use of a prespecified Lan-DeMets (O’Brien-Fleming) $\alpha$ spending function. The independent data monitoring committee reviewed the interim analysis results on 17 December 2020 and agreed that the efficacy boundary for progression-free survival had been met. Data from the prespecified interim analysis are reported in this paper and the trial is ongoing to collect long-term survival and safety data.

Efficacy was analyzed in the full analysis set, comprising all randomized patients who met the eligibility criteria, on an intention-to-treat basis. Safety was analyzed in all randomized patients who received at least one dose of the study medication. Time-to-event survival curves were estimated using the Kaplan-Meier method and treatment effects were compared using the stratified log-rank test; HRs and corresponding 95\% CIs were estimated using a stratified Cox proportional hazards model; all stratification factors were the same as those used in the randomization system. A sensitivity analysis for investigator-assessed progression-free survival was also performed, with patients experiencing a $>7-d$ delay in any treatment component due to the COVID-19 pandemic censored before treatment delay. The prespecified subgroup analysis for progression-free survival was done using an unstratified Cox proportional hazards model. The objective response and clinical benefit rates were calculated and the corresponding 95\% CIs were estimated using the Clopper-Pearson method; between-group comparisons were implemented using the stratified Cochran-Mantel-Haenszel method. Safety was summarized using descriptive statistics. All statistical analysis were performed with SAS v.9.4.

Reporting Summary. Further information on research design is available in the Nature Research Reporting Summary linked to this article.

\section{Data availability}

Individual deidentified participant data that underlie the results reported in this article will be considered for sharing after the product and indication has been approved by major health authorities (for example, China National Medical Products Administration, US Food and Drug Administration, European Medicines Agency). Data may be requested 24 months after study completion. Qualified researchers should submit a proposal to the corresponding author (bhxu@hotmail. com) outlining the reasons for requiring the data. The leading clinical site and sponsor will check whether the request is subject to any intellectual property or confidentiality obligations. Use of data must also comply with the requirements of the Human Genetics Resources Administration of China and other country- or region-specific regulations. A signed data access agreement with the sponsor is required before accessing shared data. The study protocol and statistical analysis plan are provided with the paper.

\section{Code availability}

No custom code was used for data analysis in this study.

\section{Acknowledgements}

This study was funded by Jiangsu Hengrui Pharmaceuticals (formerly Jiangsu Hengrui Medicine). This trial was designed by the principal investigator in collaboration with the sponsor. The sponsor was involved in data collection, analysis and interpretation and preparation of the manuscript. We thank all patients and their families and all members of the collaborative group in this trial. Medical writing support was provided by X. Wu (medical writer at Hengrui) according to good publication practice guidelines.

\section{Author contributions}

B.X. and J.Z. conceived and designed the study. B.X., Q.Z., P.Z., X.H., W.L., Z.T., T.S Y.T., X.Wu, Q.O., X.Y., J.C., Q.L., J.F., X.Wang, Y.Y., Y.S., Y.P., Y.W., W.X., M.Y. and Y.L. enrolled the patients and collected the data. P.Y. directed the statistical analysis and all authors participated in data interpretation. The manuscript was drafted by B.X. and P.Z. and was reviewed or revised by all authors. The final version to be submitted was approved by all authors.

\section{Competing interests}

B.X. reports receiving research grants from Hengrui, advisory fees from Novartis and Roche and fees for serving on a speakers' bureau from AstraZeneca, Pfizer, Roche and Eisai. Z.T. reports receiving research grants from Hengrui, Novartis, Bio-Thera and Eli Lilly and Company. X.Y. reports receiving research grants from Hengrui. X.Wang reports receiving research grants from Hengrui, Pfizer and Roche. X.Z., F.W., P.Y. and J.Z. were employees of Hengrui at the time of the study. The other authors declare no competing interests. 


\section{Additional information}

Extended data is available for this paper at https://doi.org/10.1038/s41591-021-01562-9.

Supplementary information The online version contains supplementary material available at https://doi.org/10.1038/s41591-021-01562-9.

Correspondence and requests for materials should be addressed to Binghe $\mathrm{Xu}$.
Peer review information Nature Medicine thanks Javier Cortes and the other, anonymous, reviewer(s) for their contribution to the peer review of this work. Javier Carmona was the primary editor on this article and managed its editorial process and peer review in collaboration with the rest of the editorial team.

Reprints and permissions information is available at www.nature.com/reprints. 


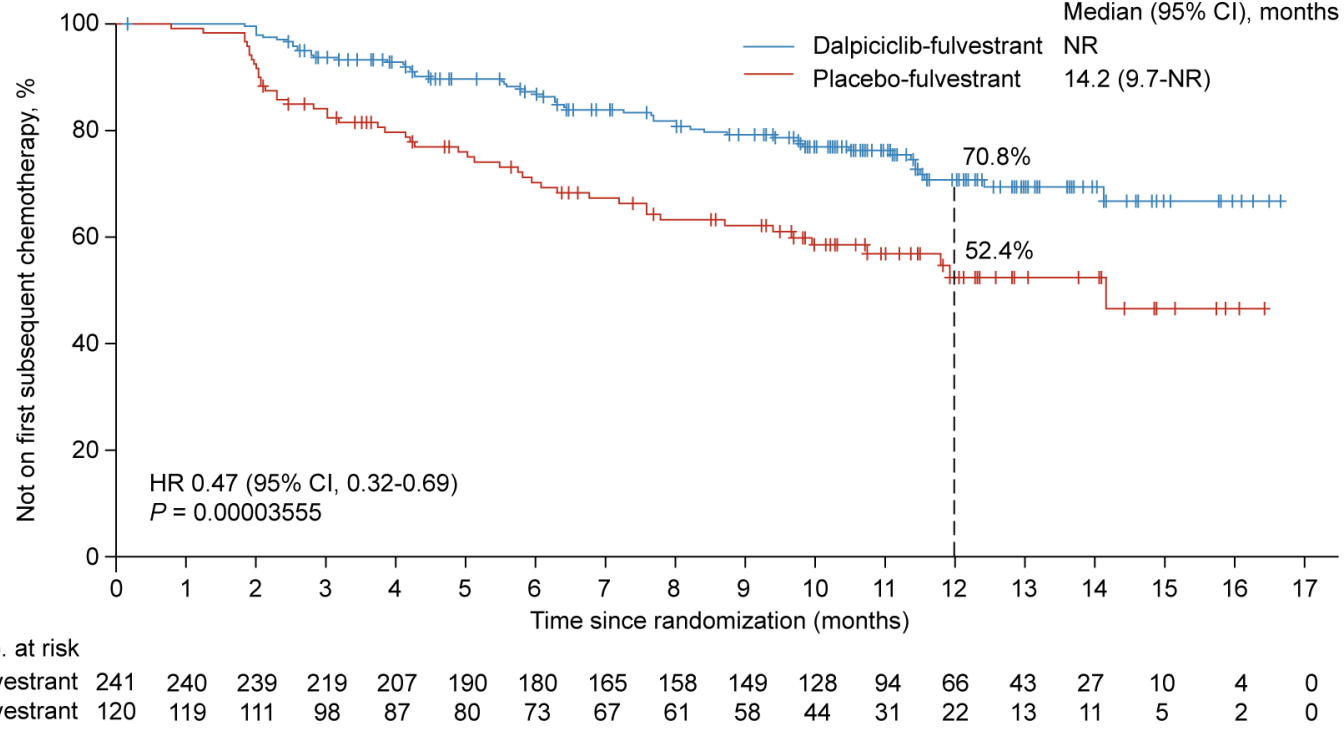

Extended Data Fig. 1 | Kaplan-Meier estimate of time to first subsequent chemotherapy or death. Treatment effects were compared using the stratified log-rank test; $\mathrm{HRs}$ and corresponding $95 \% \mathrm{Cls}$ were estimated using the Cox proportional hazards model stratified by the presence of visceral metastasis (yes vs no) and menopausal status (postmenopausal vs premenopausal or perimenopausal). The $P$ value is one-sided with no adjustment for multiplicity. $\mathrm{HR}=$ hazard ratio; $\mathrm{NR}=$ not reached. 


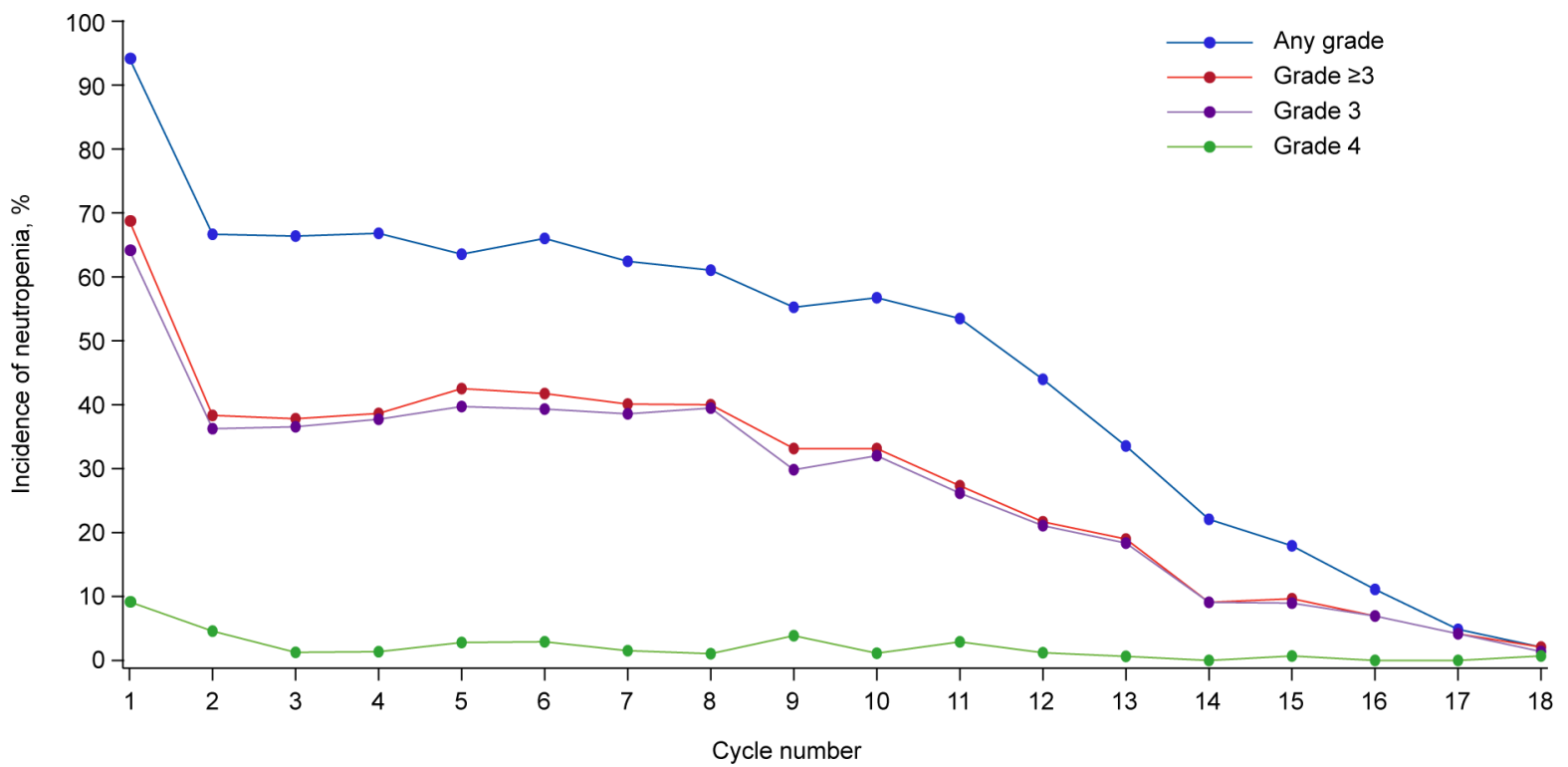

Extended Data Fig. 2 | Incidence of treatment-related neutropenia following dalpiciclib plus fulvestrant treatment. All treatment-emergent grade $\geq 3$ neutropenia were judged as treatment related by the investigators. 


\section{Reporting Summary}

Nature Portfolio wishes to improve the reproducibility of the work that we publish. This form provides structure for consistency and transparency in reporting. For further information on Nature Portfolio policies, see our Editorial Policies and the Editorial Policy Checklist.

\section{Statistics}

For all statistical analyses, confirm that the following items are present in the figure legend, table legend, main text, or Methods section.

n/a Confirmed

$\square$ The exact sample size $(n)$ for each experimental group/condition, given as a discrete number and unit of measurement

$\square$ \A statement on whether measurements were taken from distinct samples or whether the same sample was measured repeatedly

$\square$ The statistical test(s) used AND whether they are one- or two-sided

$\square$ Only common tests should be described solely by name; describe more complex techniques in the Methods section.

$\square$ \ A description of all covariates tested

$\square$ \A description of any assumptions or corrections, such as tests of normality and adjustment for multiple comparisons

$\square$ A full description of the statistical parameters including central tendency (e.g. means) or other basic estimates (e.g. regression coefficient)

$\triangle$ AND variation (e.g. standard deviation) or associated estimates of uncertainty (e.g. confidence intervals)

$\square$ For null hypothesis testing, the test statistic (e.g. $F, t, r$ ) with confidence intervals, effect sizes, degrees of freedom and $P$ value noted

Give P values as exact values whenever suitable.

\ $\square$ For Bayesian analysis, information on the choice of priors and Markov chain Monte Carlo settings

Х $\square$ For hierarchical and complex designs, identification of the appropriate level for tests and full reporting of outcomes

$\square \bigotimes$ Estimates of effect sizes (e.g. Cohen's $d$, Pearson's $r$ ), indicating how they were calculated

Our web collection on statistics for biologists contains articles on many of the points above.

\section{Software and code}

Policy information about availability of computer code

Data collection Data were collected using Medidata Rave EDC.

Data analysis All statistical analysis were performed with SAS version 9.4.

For manuscripts utilizing custom algorithms or software that are central to the research but not yet described in published literature, software must be made available to editors and reviewers. We strongly encourage code deposition in a community repository (e.g. GitHub). See the Nature Portfolio guidelines for submitting code \& software for further information.

\section{Data}

Policy information about availability of data

All manuscripts must include a data availability statement. This statement should provide the following information, where applicable:

- Accession codes, unique identifiers, or web links for publicly available datasets

- A description of any restrictions on data availability

- For clinical datasets or third party data, please ensure that the statement adheres to our policy

Individual deidentified participant data that underlie the results reported in this article will be considered for sharing after the product and indication has been approved by major health authorities (eg, China National Medical Products Administration, US Food and Drug Administration, European Medicines Agency, etc). Data may be requested 24 months after study completion. Qualified researchers should submit a proposal to the corresponding author (bhxu@hotmail.com) outlining the reasons for requiring the data. The leading clinical site and sponsor will check whether the request is subject to any intellectual property or confidentiality obligations. Use of data must also comply with the requirements of Human Genetics Resources Administration of China and other country or regionspecific regulations. A signed data access agreement with the sponsor is required before accessing shared data. The study protocol and statistical analysis plan are provided with the paper. 


\section{Field-specific reporting}

Please select the one below that is the best fit for your research. If you are not sure, read the appropriate sections before making your selection. $\bigotimes$ Life sciences $\quad \square$ Behavioural \& social sciences $\quad \square$ Ecological, evolutionary \& environmental sciences

For a reference copy of the document with all sections, see nature.com/documents/nr-reporting-summary-flat.pdf

\section{Life sciences study design}

All studies must disclose on these points even when the disclosure is negative.

Sample size

Assumptions for sample size calculation included a median progression-free survival of 6 months with placebo plus fulvestrant and 10 months with dalpiciclib plus fulvestrant and a HR of 0.60, a randomization ratio of 2:1 between two groups, an accrual period of 14 months, a duration of study period of 24 months, and a dropout rate of $15 \%$. With a one-sided significance level of $0.025,184$ PFS events were required to ensure a $90 \%$ power to detect the superiority of dalpiciclib plus fulvestrant over placebo plus fulvestrant. The sample size of 288 patients would be required. With the outbreak of the COVID-19 pandemic, the planned sample size was increased to 357 and the planned events of progression or death was increased to 227 , based on the recommendations from the independent data monitoring committee.

Data exclusions Efficacy was analyzed in the full analysis set, comprising all randomized patients who met the eligibility criteria, on an intention-to-treat basis. Safety was analyzed in all randomized patients who received at least one dose of study medication.

Replication Replication was not applicable to the study (randomized controlled trial).

Randomization Eligible patients were randomized in a 2:1 ratio to receive dalpiciclib plus fulvestrant or placebo plus fulvestrant using a central interactive web response system. Stratification factors were presence of visceral metastasis (yes vs no) and menopausal status (postmenopausal vs premenopausal or perimenopausal).

\section{Reporting for specific materials, systems and methods}

We require information from authors about some types of materials, experimental systems and methods used in many studies. Here, indicate whether each material, system or method listed is relevant to your study. If you are not sure if a list item applies to your research, read the appropriate section before selecting a response.

Materials \& experimental systems

\begin{tabular}{l|l}
\multicolumn{2}{l}{ Methods } \\
\hline n/a & Involved in the study \\
$\square$ & $\square$ ChIP-seq \\
$\square$ & $\square$ Flow cytometry \\
$\searrow$ & $\square$ MRI-based neuroimaging
\end{tabular}

$\mathrm{n} / \mathrm{a}$ Involved in the study

\ $\square$ Antibodies

\ $\square$ Eukaryotic cell lines

\ $\square$ Palaeontology and archaeology

Х

$\square$ \uman research participants

$\square \bigotimes$ Clinical data

$\bigotimes \mid \square$ Dual use research of concern

\section{Human research participants}

Policy information about studies involving human research participants

Population characteristics

Eligible patients were women aged 18-75 years, of any menopausal status (pre- or perimenopausal women were required to receive a gonadotropin-releasing hormone agonist during study), with pathologically confirmed hormone-receptor-positive, HER2-negative locally advanced or metastatic breast cancer not amenable to curative resection or radiotherapy and not indicated for chemotherapy. Patients must have had radiographically confirmed relapse or disease progression during or after previous endocrine therapy, defined as progression $\geq 6$ months after initiating endocrine therapy for recurrent or metastatic disease, or relapse or progression while on adjuvant endocrine therapy but after the first 2 years or within 12 months from completion of adjuvant endocrine therapy. Patients must not have received more than one prior chemotherapy for advanced disease. Additional inclusion criteria included an Eastern Cooperative Oncology Group performance status of 0 or 1, measurable lesion per Response Evaluation Criteria in Solid Tumors (RECIST) version 1.1 or nonmeasurable bone-only lytic or mixed lesions, adequate organ and bone marrow function, and a QT interval corrected for heart rate according to Fridericia's formula (QTCF) of $<470 \mathrm{~ms}$. The key exclusion criteria included brain metastases, prior exposure to fulvestrant, everolimus or other CDK4/6 inhibitors, significant cardiac event within 6 months, or serious infection within 4 weeks from first dose. 
Recruitment

Ethics oversight

treatment arms. With the double-blind design, all patients and investigators were masked to the treatment assigned and therefore self-selection bias was avoided.

The study protocol and all amendments were reviewed and approved by the independent ethics committee or institutional review board at each study site (National Cancer Center/National Clinical Research Center for Cancer/Cancer Hospital, Chinese Academy of Medical Sciences and Peking Union Medical College; Harbin Medical University Cancer Hospital; Fudan University Cancer Hospital; The First Hospital of Jilin University; Tianjin Medical University Cancer Institute and Hospital; Cancer Hospital of China Medical University/Liaoning Cancer Hospital; The First Affiliated Hospital of China Medical University; Hubei Cancer Hospital; Hunan Cancer Hospital; West China Hospital, Sichuan University; Union Hospital, Tongji Medical College, Huazhong University of Science \& Technology; SunYat-Sen Memorial Hospital, Sun Yat-Sen University; Jiangsu Cancer Hospital; Cancer Hospital of the University of Chinese Academy of Sciences/Zhejiang Cancer Hospital, Institute of Cancer and Basic Medicine (ICBM), Chinese Academy of Sciences; Jiangsu Province Hospital; Sun Yat-Sen University Cancer Center; Anhui Provincial Hospital; Breast Cancer Center, Shandong Cancer Hospital; Guangxi Medical University Cancer Hospital; Henan Breast Cancer Center/the affiliated Cancer Hospital of Zhengzhou University \& Henan Cancer Hospital; The Fourth Hospital of Hebei Medical University; The First Affiliated Hospital of Xiamen University; The First Affiliated Hospital of Henan University of Science \& Technology-Cancer Hospital; Southern Medical University NanFang Hospital; Tongji Hospital, Tongji Medical College, Huazhong University of Science \& Technology; The First affiliated Hospital of Guangxi Medical University; Guangdong Provincial Hospital of Traditional Chinese Medicine; The First Affiliated Hospital of Zhejiang University; Chongqing University Cancer Hospital; Anhui Provincial Cancer Hospital; The Affiliated Hospital of Qingdao University; The First Affiliated Hospital of Zhengzhou University; Ruijin Hospital Affiliated to Medical College of Shanghai Jiaotong University; The General Hospital of the People's Liberation Army; Sichuan Academy of Medical Sciences \& Sichuan Provincial People's Hospital; Zhongshan Hospital, Fudan University; The 960th Hospital of the Joint Service Support Force of the People's Liberation Army of China; The second Affiliated Hospital of Xi'an Jiaotong University; Sir Run Run Shaw Hospital ZheJiang University School of Medicine).

Note that full information on the approval of the study protocol must also be provided in the manuscript.

\section{Clinical data}

Policy information about clinical studies

All manuscripts should comply with the ICMJE guidelines for publication of clinical research and a completed CONSORT checklist must be included with all submissions.

Clinical trial registration

Study protocol

Data collection

Outcomes
ClinicalTrials.gov, NCT03927456

Submitted with manuscript

361 patients were enrolled from 39 hospitals in China between June 25, 2019 and September 2, 2020. Data cutoff date of the interim analysis was November 15, 2020. All efficacy and safety data were collected at the participating clinical centers.

The primary endpoint was investigator-assessed progression-free survival, defined as the time of randomization to the first progression per RECIST version 1.1 or death from any cause, whichever occurred first. Secondary endpoints included overall survival, progression-free survival per IRC, objective response rate, clinical benefit rate, duration of response, time to response, time to first subsequent chemotherapy or death, time to second objective disease progression (defined as time from randomization to discontinuation of next-line treatment, or tumor progression on next-line treatment or death), and safety.

Tumor evaluation was performed at screening, every 8 weeks for the first 56 weeks and every 12 weeks thereafter using computed tomography or magnetic resonance imaging by the investigator and IRC according to RECIST version 1.1. Complete or partial response was required to be confirmed with a subsequent scan at least 4 weeks after the initial documentation. Safety was monitored from the time of informed consent to 28 days after the last study dose and was assessed with adverse events, laboratory examinations and 12-lead electrocardiograms. Adverse events were graded according to the Common Terminology Criteria for Adverse Events version 5.0 . 